\title{
Estimativa da evapotranspiração em área de pastagem em Santa Maria- RS
}

Evapotranspiration estimate on pasture area in Santa Maria-RS

\author{
Gisele Cristina Dotto Rubert'ㄹ, Débora Regina Roberti², Marcelo Bortoluzzi Diaz ${ }^{1}$ e Osvaldo Luiz \\ Leal de Moraes ${ }^{2}$
}

\begin{abstract}
${ }^{1}$ Mestre, Departamento de Física, Universidade Federal de Santa Maria, Santa Maria, Brasil girubert@gmail.com; marbdiaz@gmail.com; osvaldo.moraes@gmail.com

2Doutor, Departamento de Física, Universidade Federal de Santa Maria, Santa Maria, Brasil debora@ufsm.br
\end{abstract}

\begin{abstract}
Resumo
A técnica de covariância turbulenta foi utilizada para estimar os fluxos de energia na forma de calor sensível e latente entre o Pampa gaúcho e a atmosfera no período de 20 de novembro de 2013 a 20 de novembro de 2014. Evapotranspiração (ET) acumula da anual foi de $1021 \mathrm{~mm}$, correspondendo a 55\% da precipitação. A ET apresenta alta correlação com o saldo de radiação (97\%). A razão de Bowen indica que a maior parte da energia disponível foi utilizada para a evapotranspiração. A fração evaporativa, razão entre o fluxo de energia na forma de calor latente e o saldo de radiação, não apresentou acentuada mudança sazonal, apenas uma maior variabilidade nos meses mais frios.
\end{abstract}

Palavras-chave: Bioma Pampa, Covariância de vórtices, Evapotranspiração.

\begin{abstract}
The eddy covariance technique was used to estimate the sensible and latent heat flux between the atmosphere and the Pampa Biome in the period from 20 November 2013 to 20 November 2014. Annual Evapotranspiration (ET) was $1021 \mathrm{~mm}$, corresponding to 55\% of the annual precipitation. The ET is highly correlated with the net radiation (97\%). The Bowen ratio was indicated that most of the available energy was used for the evaporation. The evaporative fraction remained about average, with greater variability in the colder months.
\end{abstract}

Keywords: Bioma Pampa, eddy covariance, Evapotranspiration. 


\section{Introdução}

A evapotranspiração (ET) é uma variável fundamental do sistema climático, sendo responsável pela conexão entre os balanços de energia e água, desempenhando assim um papel importante nas interações da biosfera-atmosferahidrosfera. Estimativas precisas da evapotranspiração entre a superfície e a atmosfera têm grande importância para a compreensão destes balanços em diferentes biomas. As regiões subtropicais constituem a maior fonte de evapotranspiração e exercem forte controle sobre os processos de circulação atmosférica. Assim, para um melhor desempenho dos modelos de circulação geral da atmosfera, da previsão de tempo e clima, dos modelos agrometeorológicos de produtividade vegetal e dos modelos hidrológicos chuva/vazão, a evapotranspiração é de fundamental importância.

Os campos do bioma Pampa estendem-se ao sul e a oeste do Uruguai e das províncias argentinas de Corrientes, Entre Rios, Santa Fé, Córdoba, Buenos Aires e La Pampa. No Brasil, o bioma Pampa ocupa a metade sul, o que corresponde a $63 \%$ da área do Estado (Figura 1). Trata-se de um bioma complexo, que possui diferentes vegetações, dentre as quais a mais representativa é o campo dominado por gramíneas. As atividades humanas têm convertido ou degradado muitas áreas deste bioma (Roesch et al., 2009). Por ser um bioma que possui fragilidade do solo, flora e fauna, um uso sustentável da terra no Pampa brasileiro é apenas possível se as atividades econômicas respeitarem as condições fornecidas pelas capacidades do solo e as adaptações de suas espécies de plantas e animais (Roesch et al., 2009). Estes fatores devem ser levados em consideração no planejamento de estratégias de conservação. Neste contexto, é fundamental a compreensão da evapotranspiração sobre este bioma. Assim, os resultados esperados para este estudo poderão servir como base de estudos futuros e aliados à modelagem podem contribuir para a nossa compreensão de como gerenciar a fim de minimizar as perdas ambientais, particularmente em face das mudanças na variabilidade de tempo e clima futuros. Neste trabalho, a estimativa da evapotranspiração utilizando a técnica de covariância de vórtices no sítio experimental de Santa Maria-RS foi analisada no período de um ano.

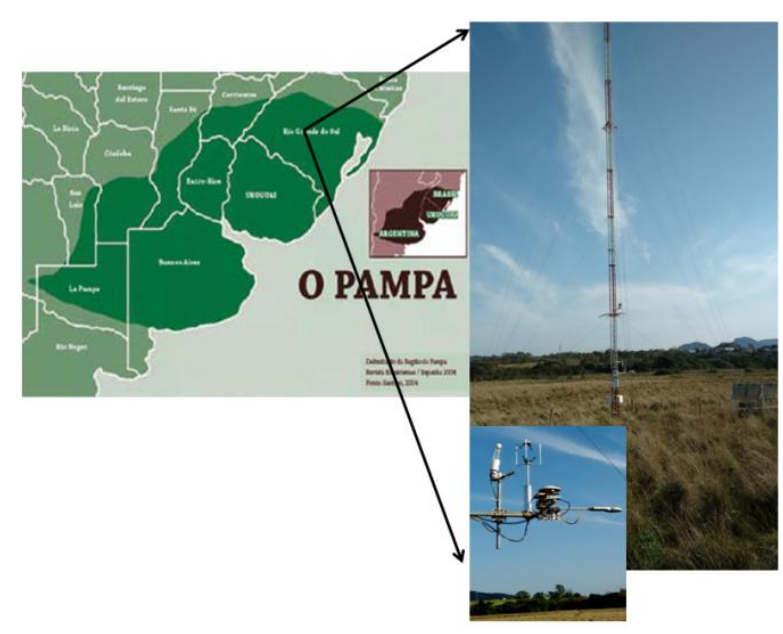

Figura 1: Abrangência do bioma Pampa, com a localização do sítio experimental de Santa MariaRS. Adaptado de: Santino, Revista

Ecossistemas/Espanha, 2004. Imagens da torre de fluxo de arquivo pessoal.

\section{Metodologia}

Os dados experimentais foram medidos em uma torre de fluxo no sítio de Santa Maria- RS (Latitude: $29^{\circ} 40^{\prime} \mathrm{S}$ e Longitude: $53^{\circ} 42^{\prime} \mathrm{W}, 88 \mathrm{~m}$ de altitude) que pertence ao Projeto Sulflux (www.ufsm.br/sulflux) do Laboratório de Micrometeorologia da Universidade Federal de Santa Maria (Figura 1). O sítio experimental encontra-se em uma área de vegetação natural no Bioma Pampa e faz parte da rede PELDCNPq (Programa de Pesquisa Ecológica de Longa Duração). O solo é classificado como Planossolo (Soil Inquérito Staff 2010). Segundo a classificação de Köppen (Nimer 1989), o clima pertence à zona do franco CFA.

A vegetação encontrada na área de estudo, utilizada como pasto para o gado de corte, é de pastagem natural com predominância de Andropogon lateralis, e outras espécies como Paspalum notatum, Saccharum trinii, Sorghastrum pellitum, e Aristida laevis. Esta composição é 
distribuída uniformemente na área do estudo (Quadros e Pilar, 2001).

A concentração de vapor de água e a velocidade do vento foram medidas a $3 \mathrm{~m}$ de altura usando um analisador de gás infravermelho caminho fechado (LI-7200, Li-Cor) e um anemômetro sônico tridimensional (Wind Master Pro/Gill Instruments). Também a $3 \mathrm{~m}$ foi medido saldo de radiação Rn (CNR4, Kipp \& Zonen), precipitação (TR525USW, Texas Eletronics. Inc.) além de temperatura e umidade relativa do ar (HMP155/ Vaisala).

Os fluxo de energia na forma de calor latente (Le) e sensível (H) foram estimados utilizando o método de covariância de vórtices com o software EddyPro ${ }^{\circledR}$, versão 5.1.1, Li-Cor (Lincoln, Nebraska, EUA). Os fluxos foram obtidos em médias de 30 minutos, com cálculo das flutuações turbulentas em média por bloco (Gash and Culf, 1996), rotação dupla (Wilczak et al., 2001) e correção para os efeitos da densidade (Webb et al., 1980). A correção espectral de alta frequência foi baseada no uso de formulações matemáticas para modelar as propriedades de fluxo e espectrais que descrevem atenuações de fluxo devido à configuração instrumental (Gash and Culf, 1996). Correções filtro passa alta e baixa seguiram a metodologia de Moncrieff et al., (2004) e Moncrieff et al., (1997), respectivamente. Testes de qualidade nos fluxos seguiram a metodologia de Foken et al., (2004). Dados faltantes de fluxo de calor latente, aproximadamente $11 \%$, foram preenchidos utilizando o método proposto por Reichstein et al., (2005). O fechamento do balanço de energia foi obtido através dos valores observados da razão de Bowen ( $\beta$ ), segundo Twine et al., (2000). Foram utilizados dados coletados no período de 20 de novembro de 2013 a 20 de novembro de 2014. A evapotranspiração diária foi calculada utilizando a média diária de Le, de acordo com a seguinte relação:

$$
E T=L e * 0,0353\left[\mathrm{mmdia}^{-1}\right]
$$

\section{Resultados}

A Figura 2 mostra a média diária a cada seis dias do fluxo de calor latente e da evapotranspiração obtidos experimentalmente sobre o Pampa gaúcho (sitio de Santa Maria). A evapotranspiração variou entre $-0,12$ e $6,86 \mathrm{~mm}$ $\mathrm{d}^{-1}$, com uma média anual de $2,78 \pm 1,63 \mathrm{~mm} \mathrm{~d}^{-1}$. A evapotranspiração acumulada no ano foi de $1021 \mathrm{~mm}$ representando $55 \%$ da precipitação observada (1862 mm ano-1). Dentre os relatos de ET para outros biomas brasileiros o bioma Pampa apresentou menores valores. Para o bioma Cerrado, a ET media anual é $1228 \mathrm{~mm}$ (Cabral et al., 2015), Goulart et al., (2015) para o Pantanal encontrou valor médio para ET de 1337,5 mm/ano. Para a Amazônia Shuttleworth (1988) obteve uma ET média anual de 1393 mm e por fim, Mata Atlântica segundo Almeida (2003) apresentou um acumulado anual de $1350 \mathrm{~mm}$. Para o pampa Argentino, Paoloni et al. (2003) obteve $1220 \mathrm{~mm}$ /ano.

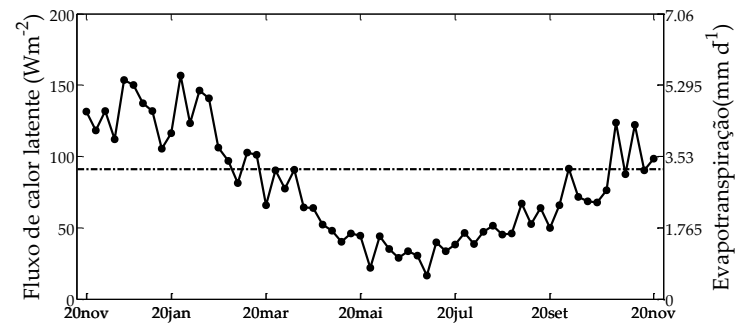

Figura 2: Fluxo de energia na forma de calor latente (eixo vertical esquerdo) e evapotranspiração (eixo vertical direito) experimental sobre o Pampa. A linha horizontal pontilhada representa a média anual. Os pontos representam média a cada 6 dias.

O fluxo de energia na forma de calor latente diminuiu com a aproximação das estações mais frias do ano, coincidindo com variações na nebulosidade e saldo de radiação (Figura 3). Os valores dos coeficientes de correlação obtidos entre Le e $\mathrm{Rn}$ foram semelhantes entre os períodos do ano analisados (outono-inverno e primevera-verão), sendo de $91 \%$ para o período outono-inverno (onde os valores para Le são menores que a média anual), 96\% no período primavera-verão (valores de Le são maiores que a média anual) e 97\% anual. Observa-se na 
Figura 3 que os menores valores para Le encontram-se no período outono-inverno, em média de $50 \mathrm{Wm}^{-2}$.

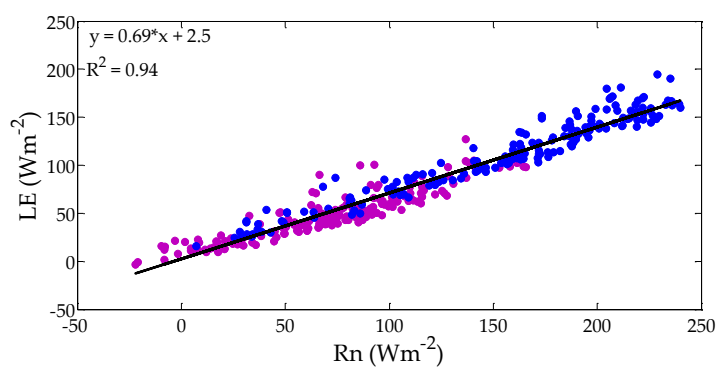

Figura 3: Diagrama de dispersão do fluxo de energia na forma de calor latente $(L e)$ em relação ao saldo de radiação durante um ano. Azul representa os valores diários para o período verão-primavera e os pontos rosa representam o período outono-inverno.

A Fração evaporativa (Le/Rn), mostrada na Figura 4, não apresentou uma acentuada variação sazonal, tendo uma maior amplitude de variação nos meses mais frios. A média anual da fração evaporativa foi de $71 \%$. Observa-se que os valores para a fração evaporativa mantêm-se dentro do desvio padrão na maior parte do ano, com valores máximo de 0,89 e mínimo de 0,54, ou seja, ao longo do ano, mais da metade da energia disponível no sistema foi utilizada para a evapotranspiração.

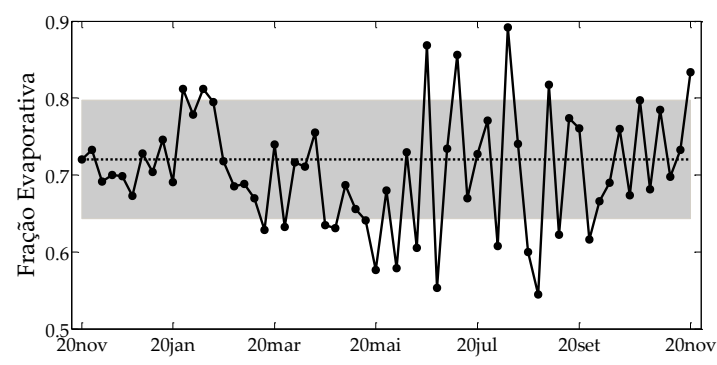

Figura 4: Fração evaporativa (Le/Rn). A linha horizontal pontilhada representa a média anual. Os pontos representam média a cada 6 dias. A área hachurada em torno da média representa o desvio padrão $(\sigma)$.

A razão de Bowen ( $\beta$ ), Figura 5, mostra o particionamento da energia disponível. Assim, podemos observar que ao longo do ano o fluxo de calor latente foi maior que o $\mathrm{H}$. No período de março a setembro observa-se uma razão de Bowen elevada, com um máximo de 1,04, representando igual partição entre H e LE.

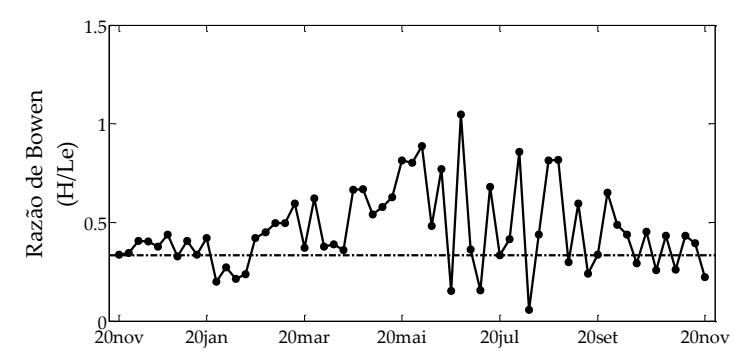

Figura 5: Razão de Bowen. A linha horizontal pontilhada representa a média anual. Os pontos representam média a cada 6 dias.

\section{Conclusão}

A quantificação da evapotranspiração é essencial para a gestão dos recursos hídricos e sua medida continua a ser um dos termos mais incertos nos balanços de água e energia. O bioma Pampa apresenta o menor valor de ET quando comparado aos demais Biomas Brasileiro e mesmo ao Pampa Argentino. Entender os processos responsáveis por este menor valor de ET é tema de pesquisas futuras.

\section{Agradecimentos}

Os autores agradecem a CAPES e ao CNPq.

\section{Referências}

Almeida, A. C., Soares, J. V. (2003). Comparação entre uso de água em plantações de eucalyptus grandis e floresta ombrófila densa (mata atlântica) na costa leste do Brasil. Revista Árvore, v.27, n.2, 159-170.

Cabral, O. M. R., da Rocha, H. R., Gash, J. H., Freitas, H. C., Ligo, M. A. V. (2015). Water and energy fluxes from a woodland savanna (Cerrado) in southeast Brazil. Jounal of Hydrology: Regional Studies 4, 22-40.

Foken, T., Gockede, M., Mauder, M., Mahrt, L., Amiro, B. D., Munger, J. W. (2004). Edited by X. 
Lee, et al. Post-field quality control, in Handbook of micrometeorology: A guide for surface flux measurements, Dordrecht: Kluwer Academic, 81-108.

Gash, J. H. C., Culf, A. D. (1996). Applying linear de-trend to eddy correlation data in real time. Boundary-Layer Meteorology, 79: 301-306.

Goulart, M. A., Sanches, L., Vilani, M. T., Pinto Júnior, O. B. (2015). Análise da evapotranspiração por wavelet de Morlet em área de Vochysia divergens Pohl no Pantanal. Revista Brasileira de Engenharia Agrícola e Ambiental, v.19, n.2, 93-98.

Moncrieff, J. B., Massheder, J. M., de Bruin, H., Ebers, J., Friborg, T., Heusinkveld, B., Kabat, P., Scott, S., Soegaard, H., Verhoef, A. (1997). A system to measure surface fluxes of momentum, sensible heat, water vapor and carbon dioxide. Journal of Hydrology, 188-189: 589-611.

Moncrieff, J. B., Clement, R., Finnigan, J., Meyers, T. (2004). Averaging, detrending and filtering of eddy covariance time series, in Handbook of micrometeorology: a guide for surface flux measurements, eds. Lee, X., W. J. Massman and B. E. Law. Dordrecht: Kluwer Academic, 7-31.

Nakai, T., Shimoyama, K. (2012). Ultrasonic anemometer angle of attack errors under turbulent conditions. Agricultural and Forest Meteorology, 18: 162-163.

Nimer, E. (1989) 'Climatologia do Brasil.' (IBGE: Rio de Janeiro).

Paoloni, J.D., Sequeira, M.E., Fiorentino, C.E., Amiotti, N.M., Vazquez, R.J. (2003). Waterresources in the semi-arid PampaPatagonia transitional region of Argentina. J.Arid Environ. 53, 257-270.

Quadros, F.L.F., Pillar V.P. (2001). Dinâmica vegetacional em pastagem natural submetida a tratamentos de queima e pastejo. Ciência Rural 31: 863-868.

Reichstein, M. et al. (2005). On the separation of net ecosystem exchange into assimilation and ecosystem respiration: review and improved algorithm. Global Change Biology, 11, 1424-1439.

Roesch, L. F. W., Vieira, F. C. B., Pereira, V. A., Schünemann, A. L., Teixeira, I. F.; Senna, A. J. T.; Stefenon, V.M. (2009). The Brazilian Pampa: A Fragile Biome. Diversity, 1, 182-198.

Santino. Revista Ecossistemas/Espanha, (2004). In:Tchê Pampa: histórias da natureza gaúcha. Cap. 3, pág 43 Campos Sulinos - conservação e uso sustentável da biodiversidade / Valério De Patta Pillar... [et al.]. Editores. - Brasília: MMA, 2009.

Soil Survey Staff (2010). Keys to Soil Taxonomy. (USDA-Natural Resources Conservation Service: Washington, DC).

Shuttleworth, W. J. (1988). Evaporation from amazonian rain forest. Proceedings of the Royal Society of London Series B 233:321-346.

Twine, T. E., Kustas, W. P., Norman, J. M., Cook, D. R., Houser, P. R., Meyers, T. P., Prueger, J. H., Starks, P. J., Wesely, M. L. (2000). Correcting eddy-covariance flux underestimates over a grassland. Agricultural and Forest Meteorology 103:279-300.

Webb, E. K., Pearman, G. I., Leuning, R. (1980). Correction of flux measurements for density effects due to heat and water vapor transfer. Quarterly Journal of the Royal Meteorological Society, 106: 85-100.

Wilczak, J. M., Oncley, S. P., Stage, S. A. (2001). Sonic anemometer tilt correction algorithms. Boundary-Layer Meteorology, 99: 127-150. 